\title{
Article \\ Clinical Utility of microRNAs in Exhaled Breath Condensate as Biomarkers for Lung Cancer
}

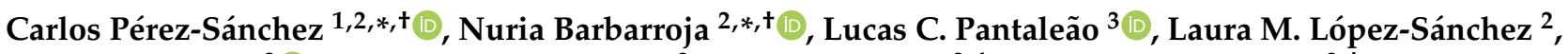 \\ Susan E. Ozanne ${ }^{3 \oplus 0}$, Bernabé Jurado-Gámez ${ }^{2}$, Enrique Aranda ${ }^{2,4}$, Chary Lopez-Pedrera ${ }^{2, \ddagger}$ \\ and Antonio Rodríguez-Ariza ${ }^{2,4, \mp(1)}$
}

check for

updates

Citation: Pérez-Sánchez, C.; Barbarroja, N.; Pantaleão, L.C.; López-Sánchez, L.M.; Ozanne, S.E.; Jurado-Gámez, B.; Aranda, E.; Lopez-Pedrera, C.; Rodríguez-Ariza, A. Clinical Utility of microRNAs in Exhaled Breath Condensate as Biomarkers for Lung Cancer. J. Pers. Med. 2021, 11, 111. https://doi.org/ 10.3390/jpm11020111

Academic Editor:

Kenneth P. H. Pritzker

Received: 21 December 2020

Accepted: 6 February 2021

Published: 9 February 2021

Publisher's Note: MDPI stays neutral with regard to jurisdictional claims in published maps and institutional affiliations.

Copyright: (c) 2021 by the authors. Licensee MDPI, Basel, Switzerland. This article is an open access article distributed under the terms and conditions of the Creative Commons Attribution (CC BY) license (https:// creativecommons.org/licenses/by/ $4.0 /)$.
1 Department of Medicine, School of Clinical Medicine, Cambridge Biomedical Campus, University of Cambridge, Cambridge CB2 0AW, UK

2 Maimonides Institute for Biomedical Research of Cordoba (IMIBIC), Reina Sofia University Hospital, University of Cordoba, 14004 Córdoba, Spain; lmlopezsanchez@gmail.com (L.M.L.-S.); bjg01co@hotmail.com (B.J.-G.); earandaa@seom.org (E.A.); charylopezpedrera@gmail.com (C.L.-P.); antonio.rodriguez.exts@juntadeandalucia.es (A.R.-A.)

3 Metabolic Research Laboratories and MRC Metabolic Diseases Unit, Welcome Trust-MRC Institute of Metabolic Science, Addenbrooke's Hospital, University of Cambridge, Cambridge CB2 0QQ, UK; lp435@medschl.cam.ac.uk (L.C.P.); seo10@medschl.cam.ac.uk (S.E.O.)

4 Centro de Investigación Biomédica en Red en Cáncer (CIBERONC), 28029 Madrid, Spain

* Correspondence: b32pesac@uco.es (C.P.-S.); barbarrojan@gmail.com (N.B.)

+ These contributed equally to this work.

$\ddagger \quad$ These contributed equally to this work.

Abstract: This study represents a novel proof of concept of the clinical utility of miRNAs from exhaled breath condensate (EBC) as biomarkers of lung cancer (LC). Genome-wide miRNA profiling and machine learning analysis were performed on EBC from 21 healthy volunteers and 21 LC patients. The levels of 12 miRNAs were significantly altered in EBC from LC patients where a specific signature of miR-4507, miR-6777-5p and miR-451a distinguished these patients with high accuracy. Besides, a distinctive miRNA profile between LC adenocarcinoma and squamous cell carcinoma was observed, where a combined panel of miR-4529-3p, miR-8075 and miR-7704 enabling discrimination between them. EBC levels of miR-6777-5p, 6780a-5p and miR-877-5p predicted clinical outcome at 500 days. Two additional miRNA signatures were also associated with other clinical features such as stage and invasion status. Dysregulated EBC miRNAs showed potential target genes related to LC pathogenesis, including CDKN2B, PTEN, TP53, BCL2, KRAS and EGFR. We conclude that EBC miRNAs might allow the identification, stratification and monitorization of LC, which could lead to the development of precision medicine in this and other respiratory diseases.

Keywords: exhaled breath condensate; microRNA; lung cancer; biomarkers; personalized medicine

\section{Introduction}

Lung cancer is the most commonly diagnosed cancer and the leading cause of cancer death worldwide, with more than 1.8 million deaths in 2018, according to a GLOBOCAN 2018 estimate [1]. The average 5-year survival rate of lung cancer is disappointingly low with a mortality rate of $85 \%$. Most patients are diagnosed at an advanced stage, and half of them will die within 1 year of diagnosis since at the time of diagnosis, only $15 \%$ of tumors are still localized allowing surgical resection. The most common type of lung cancer is non-small cell lung cancer (NSCLC), accounting for nearly $90 \%$ of all cases. The two main types of NSLC are adenocarcinoma (AD) and squamous cell carcinoma (SCC, also known as epidermoid carcinoma), which account for $30 \%$ and $25 \%$ of all lung cancers, respectively [2]. Although there have been recent advances in novel therapies for lung cancer, they are not effective at an advanced stage of the disease and, therefore, these treatments have not improved the prognosis for those patients [3]. Thus, an effective early screening 
test has been long sought to identify the disease in early stages, when treatments are much more effective. Chest radiography and sputum cytology have been tested in early screening trials, however, they were not effective in reducing lung cancer mortality rates [4]. More recently, screening with low-dose computed tomography (CT) has also been tested in several clinical trials, but data from these studies are controversial since some studies showed a reduction in mortality versus screening with standard chest X-rays $[5,6]$ while other smaller randomized controlled trials were inconclusive [7-9]. Apart from the fact that false-positive results have been commonly detected in most of these screening trials, it is also questionable whether low-dose CT could be translated into clinical practice because of the resources and expertise required for the acquisition and interpretation of images. Thus, it is crucial to develop accessible diagnostic tools to detect disease early, as well as the type, the stage and the invasion capability of the tumor. We previously identified sensitive biomarkers of lung adenocarcinoma through proteomic analysis of bronchoalveolar lavage fluid [10]. However, this requires invasive sampling so it is not suitable for the screening and early diagnosis of lung cancer. In contrast, exhaled breath condensate (EBC) contains material from the lungs and the lower respiratory tract and can be collected non-invasively. Therefore, it could be very useful in the diagnosis and prognosis of lung cancer [11]. There are several methods that can be applied to analyze the EBC for the identification of volatile compounds emitted by tumor growth, including spectrographic techniques, e-nose and trained dogs [12]. Thus, Philip et al. found a specific alteration of some volatile organic compounds (VOCs) present in EBC that could be considered as a screen for lung cancer in adult smokers [13]. Moreover, the combination of VOCs and chest CT even potentially could improve the sensitivity and specificity of lung cancer diagnosis [14].

A number of other molecules have been reported to be present in EBC, including polypeptides, proteins, and nucleic acids (DNA, mitochondrial DNA and microRNAs) [15]. In addition, mRNA isoforms of GATA6 (GATA-binding factor 6) and NKK2-1 (NK2 homeobox 1) in EBC have been proposed for lung cancer detection [16]. In this scenario, we have recently reported that the proteomic analysis of EBC samples is an appropriate approach to develop biomarkers for the diagnosis of lung cancer [17]. MicroRNAs (miRNAs) are small RNAs that by complementary sequence regulate gene expression promoting the degradation of their mRNA target genes or repressing protein translation. MiRNAs are key players in multiple cellular processes and their alteration has been associated with different diseases such as cancer [18]. The marked stability of cell-free miRNAs in body fluids and the possibility of the detection of a small number of copies represent an advantage of using miRNAs as biomarkers over other markers. Additionally, miRNAs levels in the circulation change under pathological conditions as they can be passively and actively secreted from diseased tissues. That detectable changes make cell-free miRNAs promising non-invasive biomarkers for diagnosis and disease progression.

The utility of miRNAs as biomarkers in respiratory diseases has been widely reported [19], however, their potential use as clinical tools in non-invasive samples such as EBC has not yet been fully explored.

Here we report for the first time the genome-wide miRNA expression profile in EBC samples from lung cancer patients as proof of concept of their potential clinical utility in the diagnosis, prognosis and management of respiratory diseases (study design is displayed in Figure 1). 


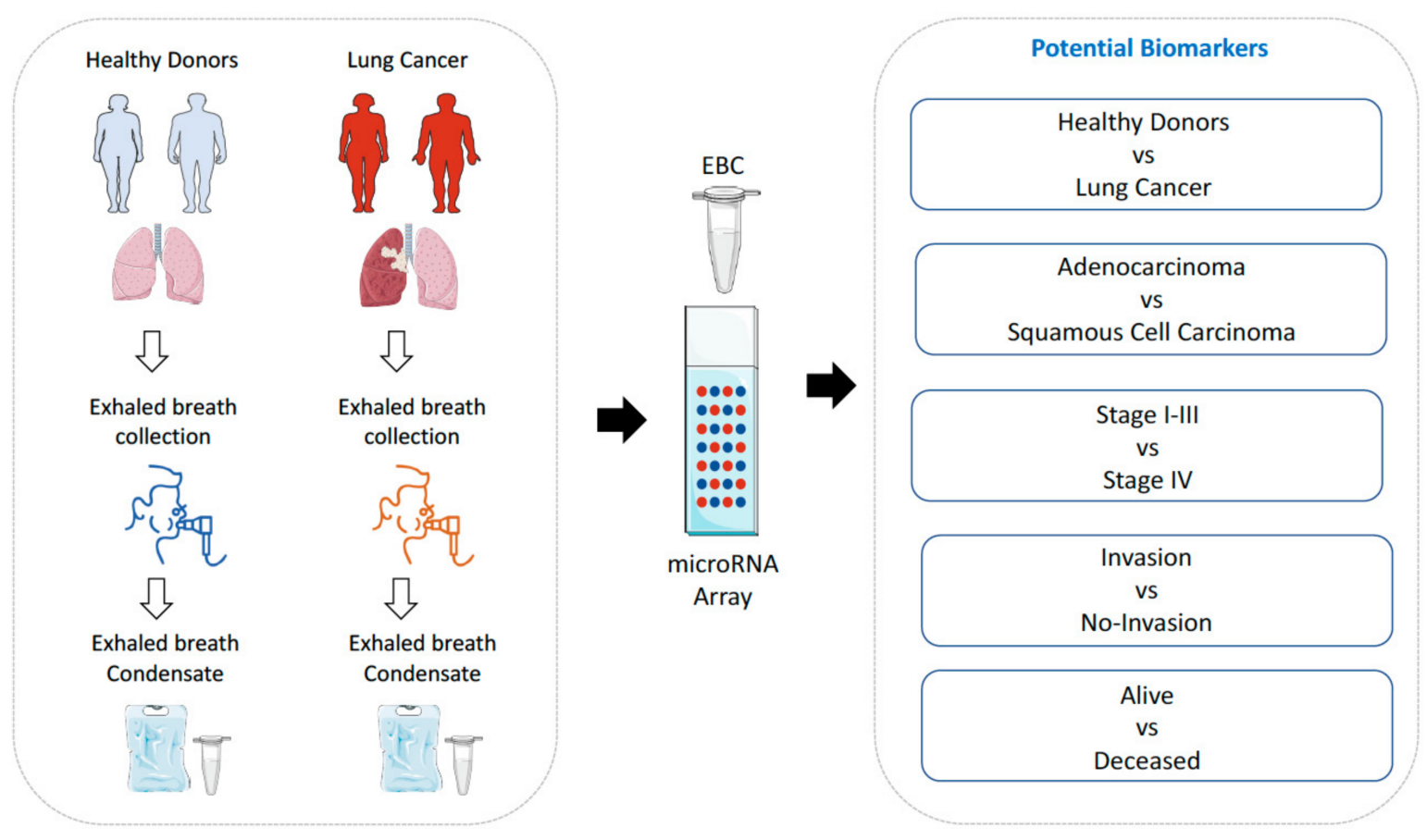

Figure 1. Overview of the study design. Genome-wide miRNA expression profiling was performed on exhaled breath condensate $(\mathrm{EBC})$ from 21 healthy volunteers and 21 lung cancer patients. The utility of EBC miRNAs as biomarkers of diagnosis, type of tumor, stage, invasion capacity and prognosis was evaluated.

\section{Materials and Methods}

\subsection{Subjects}

This is a cross-sectional study including 42 subjects from the Respiratory Medicine Department of the Reina Sofia Hospital (Córdoba, Spain).

The research was performed following the ethics code of the World Medical Association (Declaration of Helsinki). All participants signed the informed written consent. The approval of the Ethics Committee of the Reina Sofia Hospital was obtained. The EBC samples from 21 healthy donors and 21 patients diagnosed with lung cancer were collected under fasting conditions and stored at $-80^{\circ} \mathrm{C}$.

Lung cancer patients included 19 males and 2 females with an age of $62 \pm 8$ years, while healthy donors were integrated by 20 males, 1 female with an age of $61 \pm 9$ years. The type of lung cancer present in our cohort was AD (57\%) and SCC (43\%). Invasion was present in $53 \%$ of lung cancer patients, while $60 \%$ showed metastatic cancer. Stage IV was the most prevalent with 57\%, following by stage III, II and I with 24, 14 and 5\% respectively, and the tumor size was of $49 \pm 23 \mathrm{~cm}$. Clinical details of the lung cancer patients and healthy donors are displayed in Table 1.

The diagnosis included clinical tests performed on the 21 patients, based on fineneedle biopsy, bronchoscopy, video-assisted thoracoscopy and subsequent cytohistology confirmation. Subjects with coexistence of severe extrapulmonary disorder (grade IV cardiac insufficiency according to the New York Heart Association, advanced hepatic cirrhosis, stage $\mathrm{V}$ renal insufficiency), extrapulmonary neoplasm in the last five years, specific lung disease not related to smoking (including pneumonia, interstitial pneumopathy, tuberculosis, etc.) and unjustified weight loss in the last year were excluded from both groups. 
Table 1. Clinical characteristics of lung cancer patients and healthy donors.

\begin{tabular}{ccc}
\hline & Healthy Donors & Lung Cancer Patients \\
\hline Number of Subjects & 21 & 21 \\
\hline Gender (men/women) & $20 / 1$ & $19 / 2$ \\
\hline Age (mean \pm SD), years & $61.33 \pm 9.53$ & $62.85 \pm 8.06$ \\
\hline Smoking (no/yes) & $4 / 17$ & $8 / 13$ \\
\hline Lung Cancer Histology & \\
\hline Adenocarcinoma (AD) & 11 \\
\hline Squamous cell carcinoma (SCC) & 10 \\
\hline Stage (I/II/III/IV) & $1 / 3 / 6 / 11$ \\
\hline Metastasis (no/yes) & $10 / 11$ \\
\hline
\end{tabular}

\subsection{EBC Collection}

By using the EcoScreen 2 device (FILT Lungen-und Thoraxdiagnostik, Berlin, Germany) EBC was collected. Subjects wearing a nose clip breathed at a normal frequency and continuous volume for a 15-min period through a disposable mouthpiece. The system contained different valves that allowed the segregation of inhaled air from exhaled air. Thereafter, the breath was driven to a cooling device, which condensed and separated the EBC into two disposable polyethylene bags containing the EBC from the upper and the distal airways. This approach avoids saliva contamination. Besides, a protection filter (Scharlab, Barcelona, Spain) located over the inlet air valve does not allow the exogenous particles enter from the atmosphere of the room.

In this study, EBC from lower airways was used in order to reduce the contribution of upper airways (environmental factors influence) and increase the bronchial and alveolar tracts involvement.

\subsection{RNA Isolation}

RNA was isolated from lower respiratory tract EBC by using the miRNeasy Serum/ Plasma Kit (Qiagen, Hilden, Germany). This kit purifies cell-free total RNA, which primarily includes small RNAs such as miRNAs, from small volumes from diverse body fluids. In brief, two hundred microliters of EBC was mixed with denaturing buffer. Next, the manufacturer's protocols were followed for RNA extraction. Finally, RNA was recovered in $14 \mu \mathrm{L}$ of RNase-free water. The RNA concentration was quantified by NanoDrop ND-1000 (Nanodrop Technologies LLC, Wilmington, DE, USA).

\subsection{Genome-Wide miRNA Expression Profiling}

MicroRNA expression profile was carried out through GeneChipR miRNA 4.0 Array using Affymetrix technology (Affymetrix, Sunnyvale, Santa Clara, CA, USA). The platform contained 30424 probe sets designed to detect all mature human miRNA sequences (2578) included in miRBase Release 20. Microarray experiments were conducted in all samples following the manufacturer's recommendations. Shortly, $5 \mathrm{ul}$ of total RNA was labeled with FlashTag Biotin Labeling Kit and hybridized in the Affymetrix Hybridization Oven 640 at $48{ }^{\circ} \mathrm{C}$ overnight. Using fluidics script FS450_0003 the arrays were stained and then scanned on an Axon 4000B microarray scanner (Axon Instruments, Foster City, CA, USA).

Raw data were processed using oligo package for R [20]. Expression data was obtained by RNA background subtraction (treating the intensities of probe signals as a convolution of noise and true signals), quantile normalization and median-polish summarization of raw multichip data. Quality control of the output data was assessed using tests embedded into the oligo package. 


\subsection{Data Analysis and Statistics}

Prior to differential gene expression analysis, probes not designed for human miRNA detection were trimmed out from the dataset. We then selected the 5\% more abundant human miRNAs according to the individual median calculated across datasets and carried out least squares regression statistical analysis available in the limma package for $\mathrm{R}$ to identify differential gene expression between two groups [21]. Differences in miRNA expression were deemed significant when $p<0.05$. For each group comparison in this study, we also performed a principal component analysis of scaled data from regulated microRNAs. Scaled data was obtained by subtracting individual miRNA levels average from the corresponding miRNA levels of individuals samples, and by dividing the now centered results by their standard deviations. We also designed training classification models to detect variable importance amongst regulated miRNAs. A random forest algorithm was therefore applied repeatedly. At each iteration, the number of variables randomly sampled as candidates at each split in the decision trees was determined by testing the accuracy of cross-validated random forests (500 trees), and the final model was designed using the number of variables at each split that returned the best accuracy. Individual miRNA importance in the model at each interaction, indicating the mean decrease in accuracy by rearranging the values of each variable, was then identified and recorded. The least important variable was identified and removed from the next iteration until the 3 miRNAs with the highest importance were identified. We then used random forest models with leave-one-out cross-validation (LOOCV) for the seven grouping possibilities to identify the most accurate combination, and a binomial generalized linear model fitted with the selected miRNAs was generated. Finally, we determined the sensitivity and specificity of the individual selected miRNAs and the fitted model at various thresholds to generate ROC curves. We then calculated the area under the curve to determine the diagnostic ability of individual miRNAs and the fitted model to predict the outcome.

\subsection{Target Gene Prediction and Integrated Analysis by IPA}

The panel of EBC miRNAs associated with lung cancer patients and their clinical characteristics were analysed to gain insight into the biological pathways, functions and networks by using the Ingenuity Pathway Software (IPA). Potential mRNA targets (predicted with high confidence and experimentally observed) of the EBC-miRNA signature were recognised through different predicted tools (TargetScan, miRecords, TarBase, and Ingenuity ${ }^{\circledR}$ knowledge Base) (IPA, Quiagen). Through the microRNA target filter tool, we selected only mRNA targets showing either experimentally observed or high confidence predicted interactions, which have been previously associated with the pathogenesis of lung cancer.

\section{Results}

\subsection{Lung Cancer Patients Exhibit an Altered EBC miRNA Profile}

Using GeneChipR miRNA 4.0 array we profiled the miRNA expression of ECB samples from 42 subjects (21 lung cancer patients and 21 healthy controls). By applying a robust multichip average algorithm, used to subtract background, normalise quantiles and summarise raw microarray fluorescence data, the expression data from the 42 subjects included in the study was obtained. Using a multiple-linear-model approach, we identified a set of individual transcripts altered in lung cancer ( $p$-value $<0.05$ ) amongst the top $5 \%$ most abundant miRNAs. Of these, 128 miRNAs, 9 miRNAs were significantly upregulated (miR-6865-5p, miR-4707-5p, miR-451a, miR-1469, miR-4507, miR-6780a-5p, miR-668-5p, miR-6794-5p and miR-7855-5p) while 3 were downregulated (miR-3921, miR-320a, miR$6777-5 \mathrm{p}$ ) in breath condensates from lung cancer patients (Figure 2A).

A principal component analysis of scaled results from the differentially expressed miRNAs showed a separation between samples from cancer patients and healthy donors (HDs) in component 1, which explains $24.9 \%$ of the variance observed (Figure 2B). A heat 
map including the levels and clustering of those individual transcripts was also obtained (Figure 2C).

A

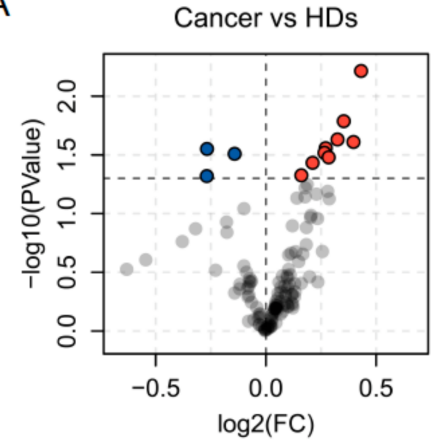

D

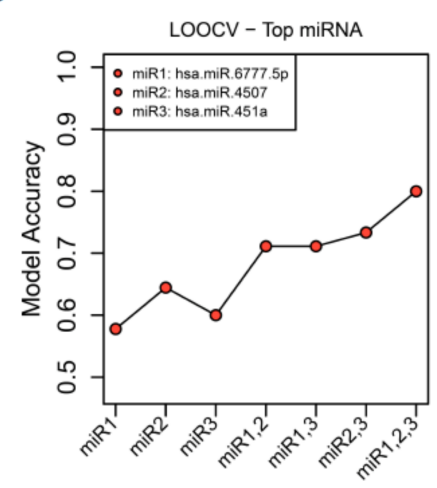

B

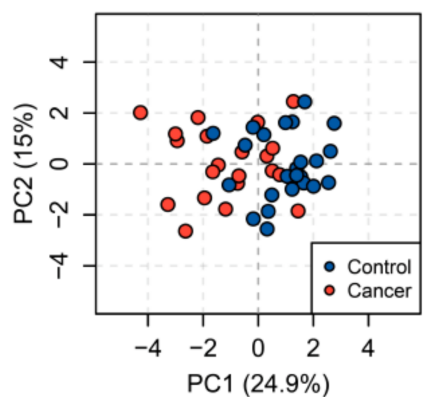

E

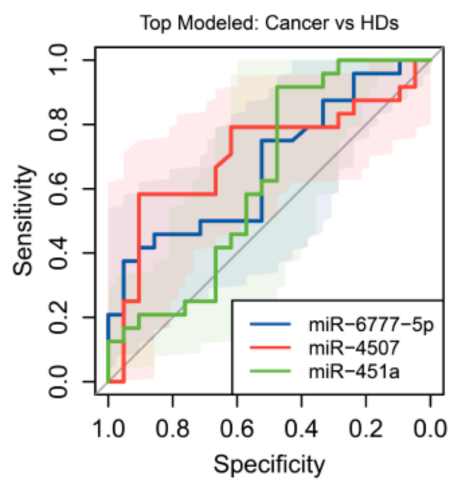

C

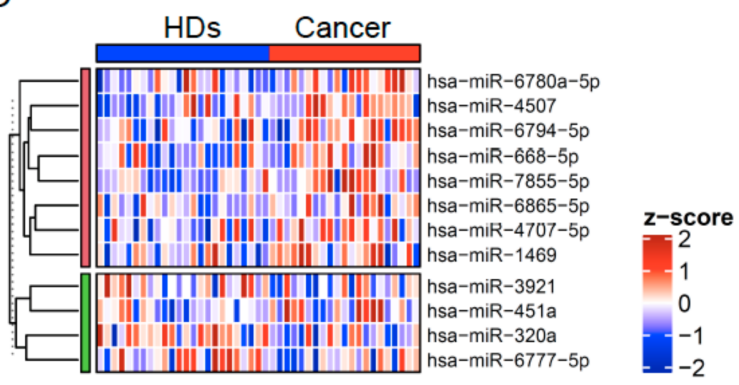

$\mathrm{F}$

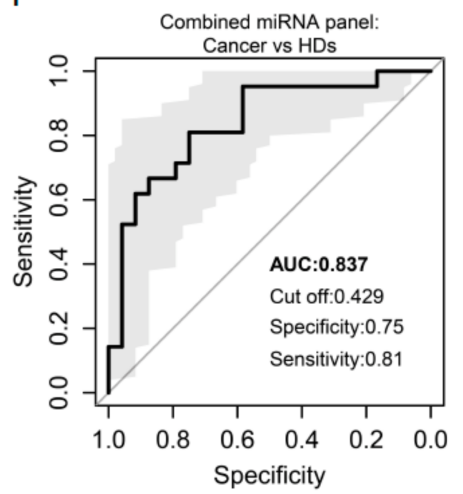

Figure 2. (A) Volcano plot showing fold change (FC) and statistical significance of individual miRNA levels in the breath condensate of cancer patients compared to healthy donors. Blue and red dots represent negatively and positively regulated miRNAs respectively $(p<0.05)$. (B) Regulated miRNAs calculated PCA plots showing the PC1 and PC2 scores for individual cancer patients (red dots) and healthy donors (blue dots). (C) Heatmap showing z-score of miRNAs regulated in healthy donors and cancer patients. (D) Accuracy of lung cancer prediction calculated by LOOCV models for individual miRNAs and for the four possible combinations. (E,F) ROC curves predicted for an individual (E) and combined miRNAs (F) selected through a random forest iteration model.

To identify the most suitable miRNAs in the breath condensate to be used as biomarkers of lung cancer, we designed training classification random forest models (500 trees) to detect variable importance. The algorithm was applied repeatedly, and at each iteration, the least important variable was flagged and removed from the next iteration until the 3 miRNAs with the highest importance were identified. We then developed random forest models with leave-one-out cross-validation (LOOCV) for the seven grouping possibilities using the selected variables and identified the model using all three miRNAs as the most accurate one (Figure 2D).

At the individual level, miR-4507 showed the best specificity and sensitivity, with the largest area under the curve (AUC of 0.70) in a receiver operating characteristic (ROC) plot followed by miR-6777-5p and miR-451a (AUC of 0.66 and 0.63, respectively) (Figure 2E). A binomial generalised linear model fitted with the three selected miRNAs was more robust and showed a higher AUC of 0.83 (Figure 2F).

\subsection{EBC miRNAs Discriminate between the Two Most Common Types of Lung Cancer AD and} SCC with High Accuracy

Twenty-eight miRNAs were differentially expressed in EBC from AD compared to SCC lung cancer patients (Figure 3A). The PCA analysis of those miRNAs might distinguish patients from both types of cancer (Figure 3B). The expression level of the signature consisting of 28 miRNAs was significantly reduced in SCC compared with AD patients 
(Figure 3C). The top three biomarker miRNAs able to distinguish AD from SCC lung cancer with the highest accuracy were miR-4529-3p, miR-8075 and miR-7704 after LOOCV and ROC curve analysis (Figure 3D,E). The combined panel integrated by those three miRNAs improved the individual capacity to discriminate between both types of lung cancer with an AUC of $0.98,100 \%$ specificity and $88 \%$ sensitivity (Figure $3 \mathrm{~F}$ ).

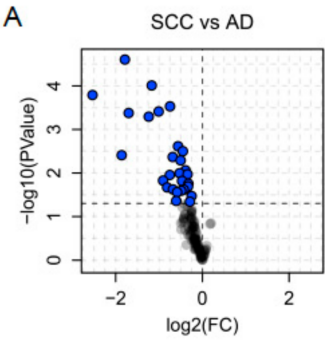

D

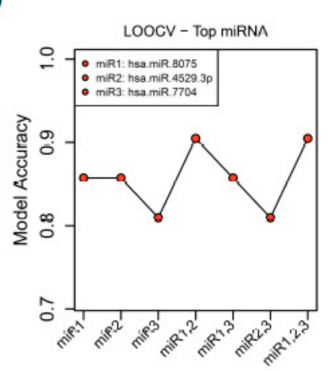

$\mathrm{E}$

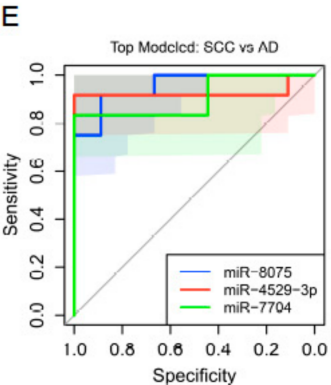

B

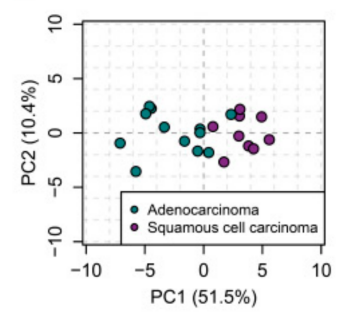

$\mathrm{F}$

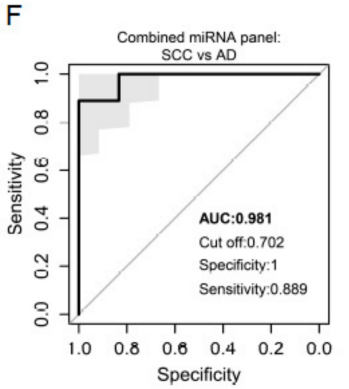

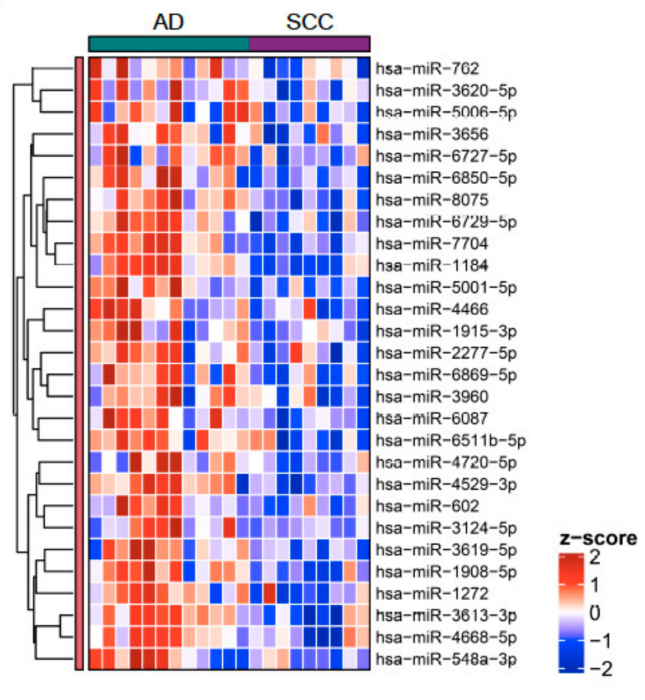

Figure 3. (A) Volcano plot showing fold change (FC) and statistical significance of individual miRNA levels in the breath condensate of SCC (squamous cell carcinoma) compared to AD (adenocarcinoma) patients. Blue dots represent negatively regulated miRNAs respectively $(p<0.05)$. (B) Regulated miRNAs calculated PCA plots showing the PC1 and PC2 scores for individual SCC patients (purple dots) and AD patients (green dots). (C) Heatmap showing z-score of miRNAs regulated in EBC from SCC and AD patients. (D) Accuracy of specific cancer type prediction calculated by LOOCV models for individual miRNAs and for the four possible combinations. (E,F) ROC curves predicted for an individual (E) and combined miRNAs $(\mathbf{F})$ selected through a random forest iteration model.

\subsection{Association of EBC miRNAs with Clinical Features of Lung Cancer: Stage and Invasion}

Three specific signatures of EBC miRNAs distinguished clinical characteristics of lung cancer including stage and invasion capability. A signature of 8 EBC miRNAs was downregulated in patients with stage IV compared to those patients with less advanced disease defined by stages I, II or III. This EBC miRNA signature was composed of miR-548ae, miR-548ac, miR-1272, miR-4529-3p, miR-3124-5p, miR-602, miR-4787-5p and miR-551b$5 p$ (Figure $4 \mathrm{~A}-\mathrm{C}$ ). The best EBC miRNA candidates as biomarkers to identify patients with the most advanced stage of the disease were miR-602, miR-551b-5p and miR-1272 and the combined panel of these three miRNAs improved the discrimination capacity of each miRNA independently with an AUC of $0.88,89 \%$ specificity and $92 \%$ sensitivity (Figure 4D-F).

Regarding the invasion capability of the tumors, a signature of 9 EBC miRNAs was differentially expressed between invasive and non-invasive tumors. Invasive tumors were characterised by the downregulation of miR-1233-5p, miR-6729-5p, miR-1298-3p, miR-548x3p, miR-548a-3p, miR-4668-5p, miR-663a, miR-1272, and the upregulation of miR-6803-5p (Figure 5A-C). Three of these EBC miRNAs showed the highest accuracy as biomarkers of invasion in lung cancer tumors including miR-6803-5p, miR-548x-3p and miR-1272. The combination of these miRNAs in an integrated panel distinguished invasive from non-invasive tumors with $100 \%$ specificity and sensitivity in our cohort (Figure 5D-F). 


\subsection{EBC miRNAs Predict the Clinical Outcome of Lung Cancer Patients with High Accuracy}

In order to evaluate the capacity of EBC miRNAs as biomarkers of prognosis, we performed a prospective clinical follow-up of all the patients included in this study for 500 days since the first EBC sample was collected. The final clinical outcome of lung cancer patients after this time was defined as deceased or alive. Ten lung cancer patients were deceased and 8 were alive after 500 days. At this point, data from 3 patients were missing. Among the deceased patients, $80 \%$ were at stage IV and $20 \%$ were at stage I-III at the moment of EBC sample collection. In contrast, $25 \%$ of the alive patients were at stage IV and $75 \%$ were at stage I-III by the time of EBC testing.

A signature of three miRNAs was differentially expressed at baseline between the two prognosis groups. The expression level of miR-6777-5p was up-regulated, while 6780a-5p and miR-877-5p were downregulated in the deceased group compared with the alive group after 500 days. A principal component analysis highlighted the stratification of both prognosis groups based on the expression of these miRNAs (Figure 6A-C). The potential of the EBC miRNA signature as biomarker predictors of prognosis in lung cancer patients was demonstrated through the ROC curve analysis which showed individual AUCs above 0.8 . The combined model integrated by those three miRNAs predicted the prognosis at 500 days with $100 \%$ specificity and sensitivity along with an AUC of 1 in our cohort of patients (Figure 6D-F). Interestingly, as 20-25\% of lung cancer patients did not show a correlation between their stage and outcome at 500 days, the panel of miRNAs predictors of each clinical feature was independent.

A

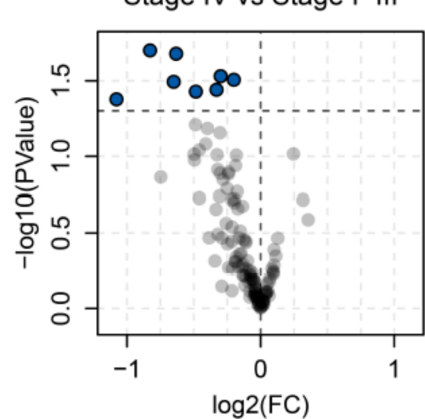

D

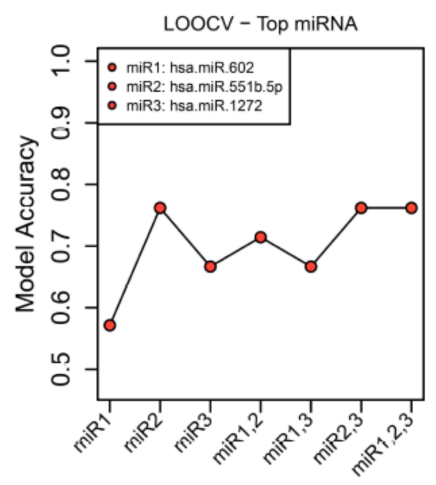

B

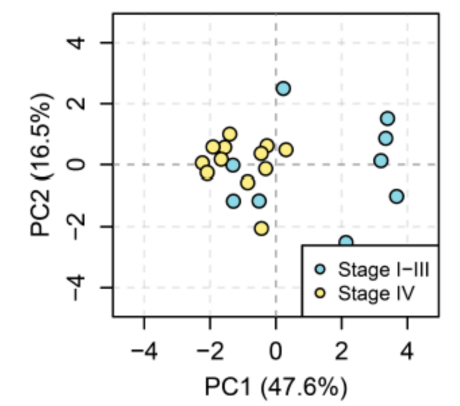

E

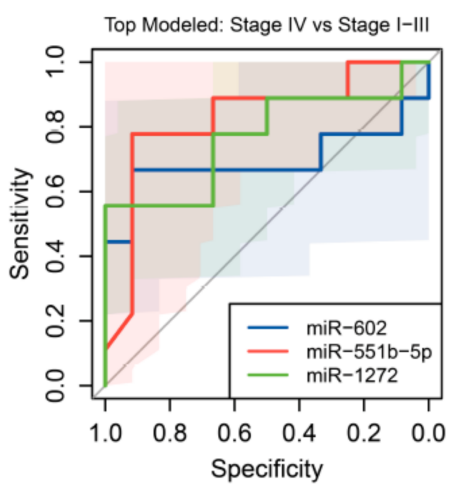

C

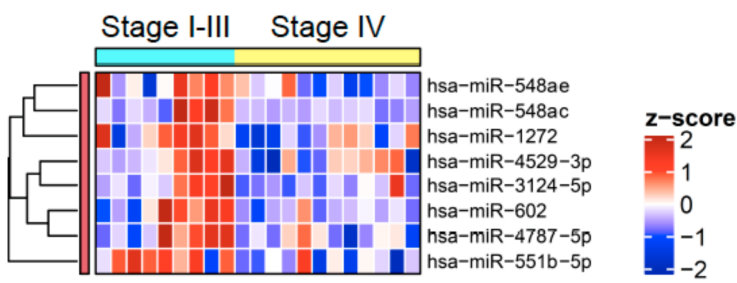

F

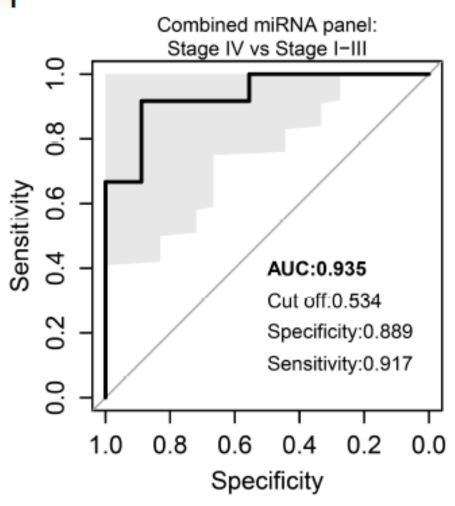

Figure 4. (A) Volcano plot showing fold change (FC) and statistical significance of individual miRNA levels in the breath condensate of lung cancer patients with stage IV compared to stage I-III. Blue dots represent negatively regulated miRNAs $(p<0.05)$. (B) Regulated miRNAs calculated PCA plots showing the PC1 and PC2 scores for individual lung cancer patients with stage IV (yellow dots) and stage I-III (blue dots). (C) Heatmap showing z-score of miRNAs regulated in EBC from lung cancer patients with stage I-III and stage IV. (D) Accuracy of stage prediction calculated by LOOCV models for individual miRNAs and for the four possible combinations. (E,F) ROC curves predicted for an individual (E) and combined miRNAs (F) selected through a random forest iteration model. 
A

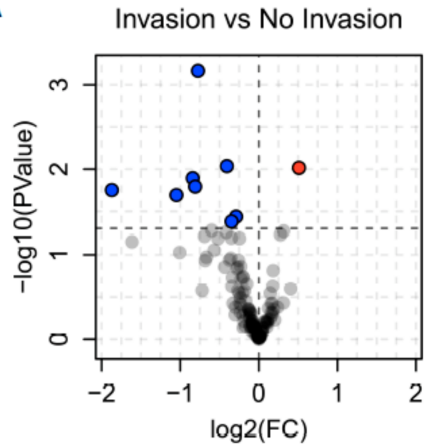

D

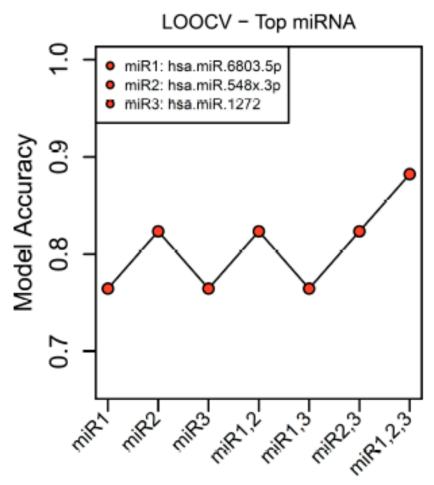

B

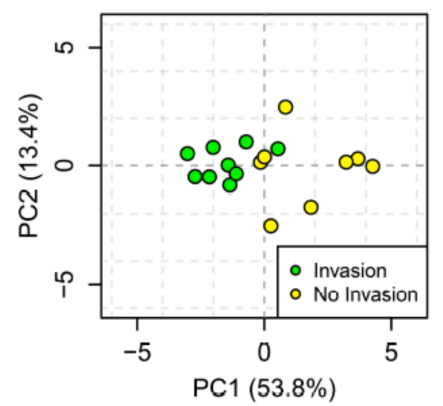

E

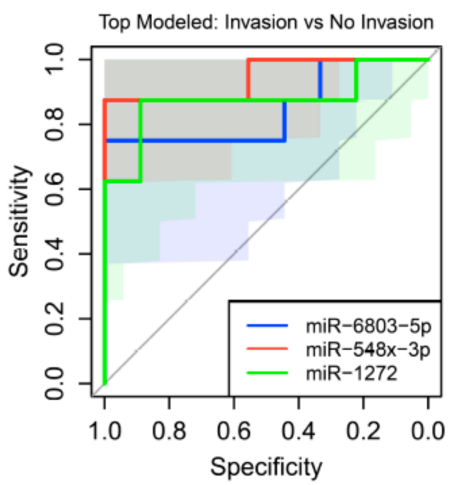

C

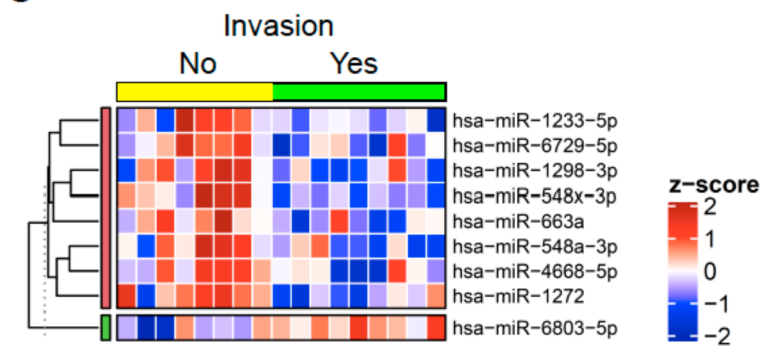

$\mathrm{F}$

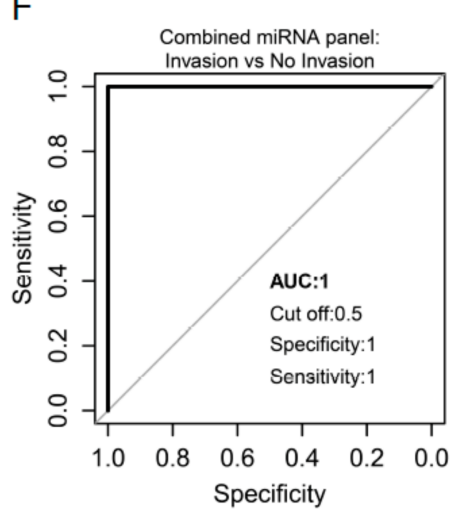

Figure 5. (A) Volcano plot showing fold change (FC) and statistical significance of individual miRNA levels in the breath condensate of lung cancer patients with invasion compared to those having no invasion. Blue and red dots represent negatively and positively regulated miRNAs respectively $(p<0.05)$. (B) Regulated miRNAs calculated PCA plots showing the PC1 and PC2 scores for individual lung cancer patients with invasion (green dots) and patients having no invasion (yellow dots). (C) Heatmap showing z-score of miRNAs regulated in EBC from lung cancer patients with and without invasion. (D) Accuracy of invasion capacity prediction calculated by LOOCV models for individual miRNAs and for the four possible combinations. (E,F) ROC curves predicted for an individual (E) and combined miRNAs (F) selected through a random forest iteration model.

Taken together, our results highlight the potential of several miRNAs as useful biomarkers candidates for diagnosis, prognosis and clinical features of lung cancer (Table 2).

\subsection{The EBC miRNA Signature Associated with Lung Cancer and Its Clinical Features Shows Multiple Potential mRNA Targets Related to the Disease}

Ingenuity Pathway Analysis allowed us to identify potential targets of the miRNAs identified in EBC that were signatures of lung cancer and associated with its clinical features. This included a remarkable number of potential targets known to be related to the pathogenesis of the disease. Thus, a complex network between EBC miRNAs and lung cancer-associated mRNA targets was obtained where individual miRNAs simultaneously interact with multiples mRNA targets. Among these miRNAs, miR-5001-5p, $-6780 a-5 p$, $-6794-5 p,-5006-5 p,-1908-5 p,-1233-5 p,-6803-5 p,-6865-5 p$, and miR-6777-5p showed more than 10 potential lung cancer-associated mRNA targets at the same time (Figure 7A). Reciprocally, in this network, a number of individual lung cancer-associated mRNA targets could be simultaneously regulated by multiples EBC miRNAs. Among these lung cancerassociated genes, CDKN2B, CYCS, BCL2L1, CDK6, PTEN, TP53, BCL2, CDKN2A, IKBKG, RASD1, STK4, TGFA and TRAF1, were predicted to be regulated by more than 5 EBC miRNAs at the same time (Figure $7 \mathrm{~B}, \mathrm{C}$ ). 
A

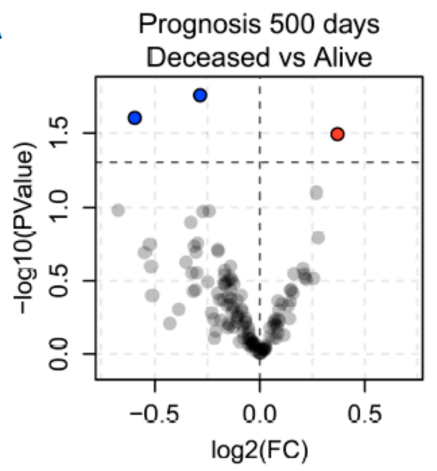

D

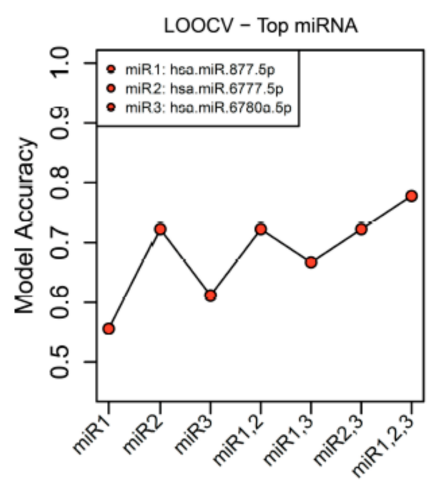

B

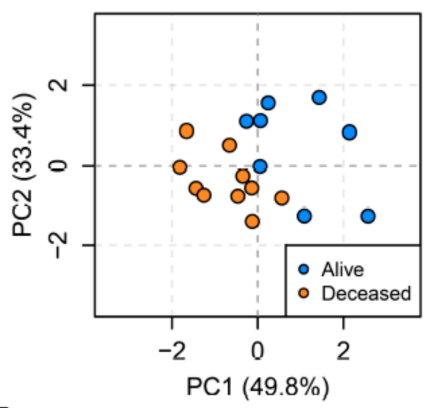

E

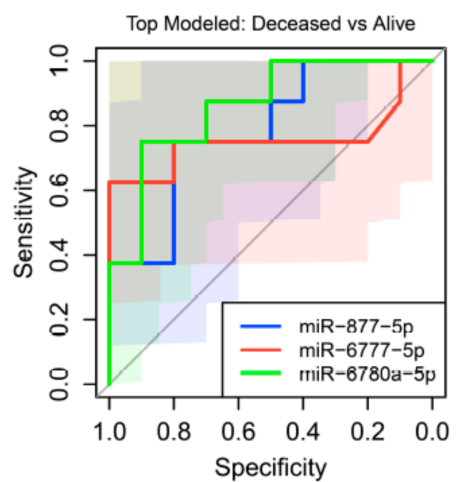

C

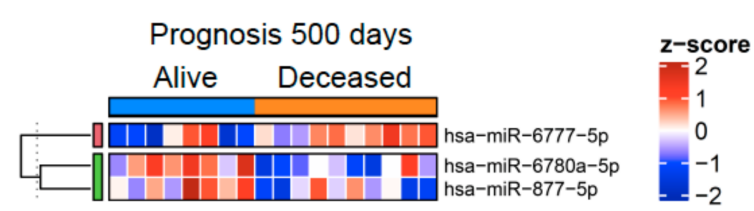

F

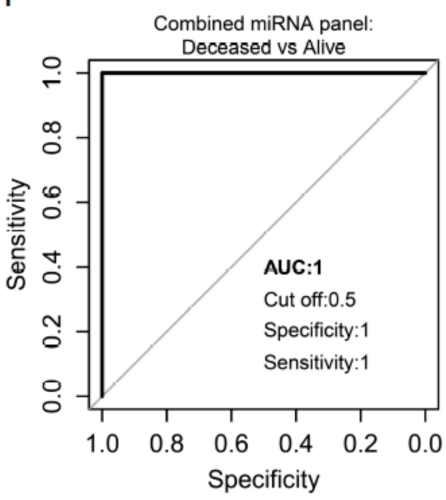

Figure 6. (A) Volcano plot showing fold change (FC) and statistical significance of individual miRNA levels in the breath condensate of lung cancer patients who were deceased after 500 days and those alive. Blue and red dots represent negatively and positively regulated miRNAs respectively $(p<0.05)$. (B) Regulated miRNAs calculated PCA plots showing the PC1 and PC2 scores for individual lung cancer patients alive (blue dots) and deceased (orange dots). (C) Heatmap showing z-score of miRNAs regulated in EBC from lung cancer patients who died and are alive after 500 days. (D) Accuracy of invasion capacity prediction calculated by LOOCV models for individual miRNAs and for the four possible combinations. (E,F) ROC curves predicted for an individual (E) and combined miRNAs (F) selected through a random forest iteration model.

Table 2. EBC miRNAs with potential as biomarkers in lung cancer.

\begin{tabular}{cccccc}
\hline \multicolumn{2}{c}{ Cancer vs. HDs } & \multicolumn{2}{c}{ Type of Cancer: AD vs. SCC } & \multicolumn{2}{c}{ Stage I-III vs. Stage IV } \\
\hline EBC miRNAs & Combined Model & EBC miRNAs & Combined Model & EBC miRNAs & Combined Model \\
\hline miR-6777-5p & AUC: 0.837 & miR-8075 & AUC: 0.981 & miR-602 & AUC: 0.935 \\
miR-4507 & Specificity: 0.75 & miR-4529-3p & Specificity: 1 & miR-551b-5p & Specificity: 0.88 \\
miR-451a & Sensitivity: 0.81 & miR-7704 & Sensitivity: 0.88 & miR-1272 & Sensitivity: 0.91 \\
\hline \multicolumn{4}{c}{ Invasion vs. No Invasion } & Prognosis 500 days: Deceased vs. Alive \\
\hline & EBC miRNAs & Combined Model & EBC miRNAs & Combined Model \\
\hline & miR-6803-5p & AUC: 1 & miR-877-5p & AUC: 1 \\
miR-548x-3p & Specificity: 1 & miR-6777-5p & Specificity: 1 \\
& miR-1272 & Sensitivity: 1 & miR-6780a-5p & Sensitivity: 1 \\
\hline
\end{tabular}


A

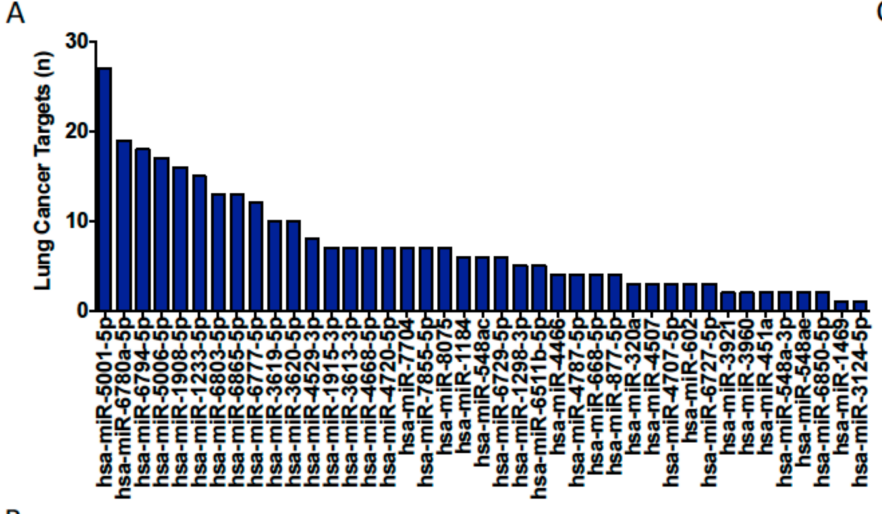

B

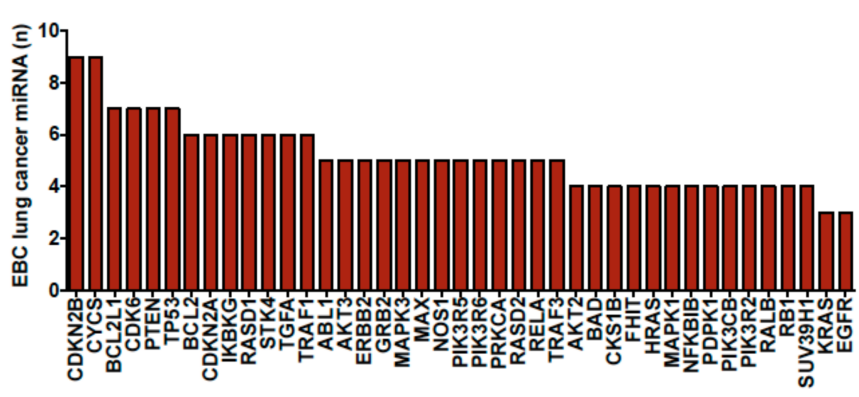

c

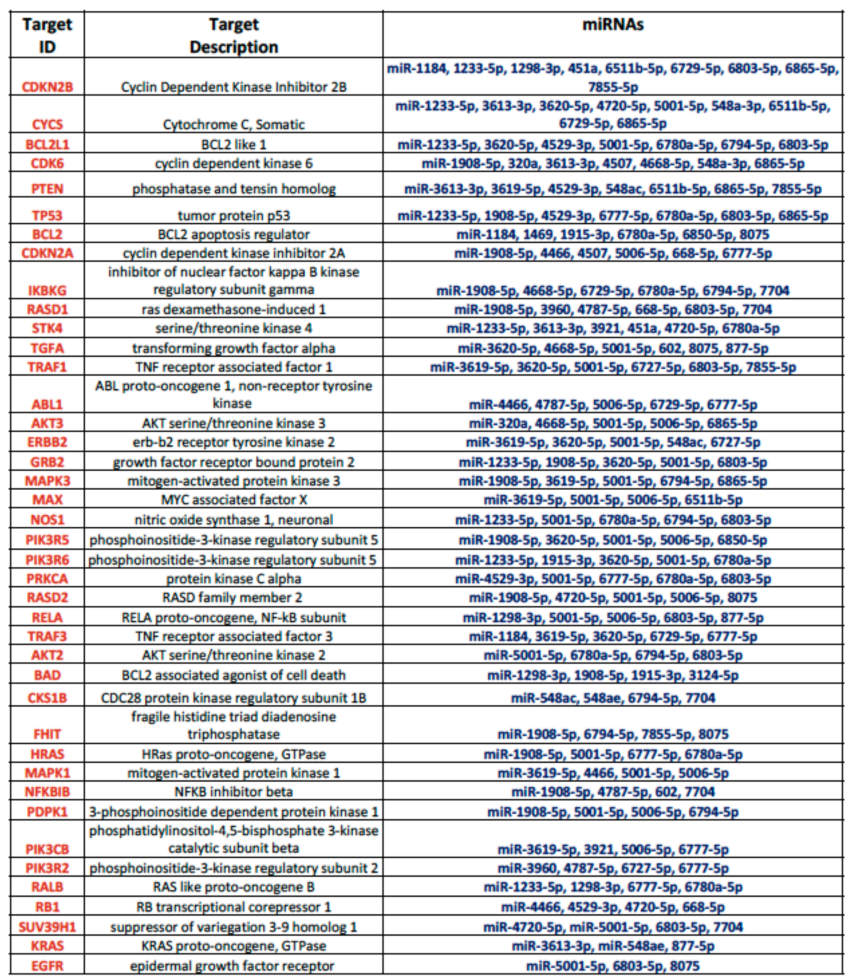

Figure 7. (A) Number of potential mRNA targets of the miRNAs identified in EBC that were signatures of lung cancer and associated with its clinical features. Individual miRNAs can simultaneously interact with multiples mRNA targets. (B,C) A number of individual lung cancer-associated mRNA targets could be simultaneously regulated by multiples EBC miRNAs.

\section{Discussion}

We here show the proof of concept of the clinical utility of miRNAs from EBC as non-invasive biomarkers for lung cancer. Previous studies have shown the potential of EBC miRNAs as biomarkers for other respiratory diseases such as asthma, Chronic Obstructive Pulmonary Disease (COPD), and pulmonary tuberculosis [22-24]. Pinkerton et al. analysed a candidate panel of 39 miRNAs by PCR in 11 patients with asthma, 10 with COPD and 12 healthy volunteers (HDs). Three miRNAs including miR-1248, miR-1291 and let-7 were downregulated in asthma compared with COPD and HDs, while miR-328 and miR-21 were lower in both respiratory diseases compared with HDs [22]. Sinha et al. also analysed a larger candidate panel of miRNAs in EBC from 10 patients with asthma and $10 \mathrm{HDs}$ by PCR. Seven miRNAs (miR-649, 1264, 2861, 574-5p, 453, 4256, 556-5p) were simultaneously altered in both asthma and pulmonary tuberculosis, although to a much higher degree in the tuberculosis group. They demonstrated that most of the detected miRNAs in EBC were present in exosomes allowing the transference of miRNAs between cells and increasing their stability in this biofluid [23]. To date, the only previous study of EBC in relation to lung cancer, was by Monzzoni et al. who analysed the levels of two selected miRNAs in EBC and plasma from 54 NSCLC and 46 HDs by PCR. They showed upregulation of miR-21 and downregulation of miR-486 in both EBC and plasma from lung cancer patients, suggesting the clinical utility of miRNAs as biomarkers for the diagnosis of NSCLC [24].

Our data showed that the unsupervised analysis of the whole miRNome in EBC allows the identification of miRNA signatures that might differentiate individuals with lung cancer. New studies with large cohorts of patients are needed to replicate and validate these and other models that would significantly improve the early detection and management of this and other respiratory diseases.

Currently, the only way to diagnose the type of lung cancer is through invasive biopsy techniques. This is a critical step for the clinical decision of selecting the most suitable 
therapy for each patient. Several studies have identified potential miRNAs candidates as biomarkers for typifying the type of cancer in histological samples. For example, Zhang et al. identified four miRNAs highly expressed in SCC including miR-93, miR-205, miR-221 and miR-30e, while adenocarcinoma was characterised by high expression of five miRNAs like miR-29b, let-7e, miR-100, miR-29c and miR-125a-5p [25]. The specificity of miR-205 as biomarker of SCC in lung biopsies has been confirmed in other two additional studies [26,27]. In adenocarcinoma, Nadal et al. showed that miR-411, miR-370 and miR$376 a$, were associated with poor survival after resection [28]. The translational potential of these histological biomarkers was also demonstrated by the development of a microRNAbased diagnostic assay called "miRview lung", which distinguished four main lung cancer subtypes with high accuracy (94\%) using the expression of a signature of 8 miRNAs (miR205, miR-21, miR-125a-5p, miR-29b, miR-106a, miR-129-3p, miR-7, and miR-375) [29]. In our study, we showed for the first time, that miRNAs coming directly from the respiratory tract in non-invasive samples such as EBC can also identify the type of lung cancer with a high degree of accuracy in a small cohort of patients. These promising results open the door for new clinical studies to confirm the clinical utility of this non-invasive test which might influence the early diagnosis, treatment and quality of life of these patients.

Despite the standard therapies, the severity and rapid progression of lung cancer are associated with the aggressive behaviour of the disease involving key processes such as invasion, migration and epithelial-to-mesenchymal transition. Numerous studies have shown the key role of miRNAs in the regulation of genes, including transcription factors and signalling pathways related to epithelial cell plasticity, cell invasion, migration and metastasis [30]. The search for biomarkers that contribute to the early identification of these processes is a crucial step in the prevention and treatment of lung cancer patients. Our pilot study demonstrated that the analysis of the expression level of miRNAs in biofluids from the respiratory tract such as EBC has the potential not only to identify the presence and the subtype of lung cancer but also clinical features such as stage, invasion and metastasis.

Most lung cancer patients are diagnosed at late stages and conventional treatments including surgery, chemotherapy and radiotherapy are not effective for those stages, which is translated into a poor survival rate in the short-term. Thus, early diagnosis and accurate prognosis are important factors to improve the survival rate of these patients. There are several cancer-associated miRNAs that can predict the prognosis of lung cancer [31]. A recent meta-analysis carried out by Xiao et al. which included 15 studies and 1753 lung cancer patients in total, identified a signature of 18 miRNAs (8 down and 10 up) from biopsies, serum and plasma associated with poorer overall survival [32]. In the present study, we showed for the first time that the analysis of miRNAs in EBC from lung cancer patients has the capacity to predict the clinical outcome of these patients in non-invasive samples. Thus, the identification and validation of robust miRNA signatures in EBC in large longitudinal studies might constitute a novel valuable tool for the early identification of patients at higher risk.

Interestingly, we also noticed that several miRNAs including miR-1272, miR-6858, miR-5094, miR-6727, miR-320b and miR-548 family were simultaneously associated with several clinical features, such as the presence of the tumor (miR-6858 and miR-5094), type of lung cancer (miR-1272, miR-6858 and miR-6727), metastasis (miR-1272, miR-5094, miR6727, miR-320b and miR-548 family), invasion (miR-1272 and miR-548 family) and stage (miR-1272, miR-5094, miR-320b and miR-548 family), which reinforces their relevant role as potential biomarkers of this disease.

In addition, we identified potential mRNA targets of the EBC miRNAs known to be related to lung cancer pathogenesis. Thus, individual miRNAs could simultaneously interact with multiples mRNA targets and reciprocally individual mRNA targets could be simultaneously regulated by multiples EBC miRNAs

Among them, KRAS, which is frequently mutated in lung cancer, and EGFR, which is a major therapeutic target in this disease, were also predicted targets of three miRNAs identified in EBC from lung cancer patients. 
These results highlighted the likely coordinated dysregulation of the EBC miRNA profile in lung cancer patients which might influence the pathologic mechanism driven by their potential mRNA targets. Future studies are needed to further characterise the relationship between these miRNAs and mRNA targets and to gain insight into the biologic role of miRNAs in EBC.

Limitations of the study: authors recognize the impact of the small sample size may have on the strength of the results, not allowing some kind of analysis such as the effect of the medications or the differentiation among all the stages of the tumor or other clinical features. A larger cohort of patients and healthy donors would be necessary to replicate and validate these results and reach a conclusive model.

In conclusion, our findings demonstrate proof of principle that the integration of unsupervised high-throughput analysis such as the genome-wide miRNA profiling of EBC from lung cancer patients, and advances in computational tools, such as machine learning, could allow the identification, stratification and monitorisation of lung cancer patients with high accuracy in a non-invasive type of sample. This proof of concept paves the way for the development of large clinical studies involving this and other respiratory diseases in the era of personalised medicine that could have a substantial impact on the prognosis of individuals with lung disease.

Author Contributions: C.P.-S., N.B., C.L.-P., and A.R.-A. designed the study. C.P.-S., N.B., L.C.P., and L.M.L.-S. analysed data, performed in vitro analysis and created the figures. E.A. and B.J.-G. contributed to sample collection and clinical data generation. S.E.O., C.L.-P. and A.R.-A. provided intellectual contributions to analyses. C.P.-S. and N.B. wrote the manuscript. All authors edited the manuscript. All authors have read and agreed to the published version of the manuscript.

Funding: This study was supported by the Spanish Ministerio de Economía y Competitividad and Fondo Europeo de Desarrollo Regional program.

Institutional Review Board Statement: The study was approved by the Ethics Committee at the Reina Sofia University Hospital (Protocol “ONCOVER).

Informed Consent Statement: Informed consent was obtained from all subjects involved in the study.

Data Availability Statement: The datasets used and/or analyzed during the current study are available from the corresponding authors on reasonable request.

Acknowledgments: The authors would like to thank all of the patients who participated in the study.

Conflicts of Interest: The authors declare no conflict of interest.

\section{References}

1. Bray, F.; Ferlay, J.; Soerjomataram, I.; Siegel, R.L.; Torre, L.A.; Jemal, A. Global cancer statistics 2018: GLOBOCAN estimates of incidence and mortality worldwide for 36 cancers in 185 countries. CA Cancer J. Clin. 2018, 68, 394-424. [CrossRef] [PubMed]

2. Herbst, R.S.; Heymach, J.V.; Lippman, S.M. Molecular origins of cancer: Lung cancer. N. Engl. J. Med. 2008, 359, 1367-1380. [CrossRef]

3. Ho, J.C.M.; Tam, T.C.C.; Lam, S.K. Salvage therapy beyond targeted therapy in lung adenocarcinoma. Semin. Respir. Crit. Care Med. 2013, 34, 837-844. [CrossRef] [PubMed]

4. Berlin, N.I.; Buncher, C.R.; Fontana, R.S.; Frost, J.K.; Melamed, M.R. The National Cancer Institute Cooperative Early Lung Cancer Detection Program. Results of the initial screen (prevalence). Early lung cancer detection: Introduction. Am. Rev. Respir. Dis. 1984, 130, 545-549. [CrossRef]

5. The National Lung Screening Trial Research Team. Reduced Lung-Cancer Mortality with Low-Dose Computed Tomographic Screening. N. Engl. J. Med. 2011, 365, 395-409. [CrossRef]

6. De Koning, H.J.; van der Aalst, C.M.; de Jong, P.A.; Scholten, E.T.; Nackaerts, K.; Heuvelmans, M.A.; Lammers, J.-W.J.; Weenink, C.; Yousaf-Khan, U.; Horeweg, N.; et al. Reduced Lung-Cancer Mortality with Volume CT Screening in a Randomized Trial. N. Engl. J. Med. 2020, 382, 503-513. [CrossRef] [PubMed]

7. Infante, M.; Cavuto, S.; Lutman, F.R.; Brambilla, G.; Chiesa, G.; Ceresoli, G.; Passera, E.; Angeli, E.; Chiarenza, M.; Aranzulla, G.; et al. A randomized study of lung cancer screening with spiral computed tomography: Three-year results from the DANTE trial. Am. J. Respir. Crit. Care Med. 2009, 180, 445-453. [CrossRef]

8. Pastorino, U.; Rossi, M.; Rosato, V.; Marchianò, A.; Sverzellati, N.; Morosi, C.; Fabbri, A.; Galeone, C.; Negri, E.; Sozzi, G.; et al. Annual or biennial CT screening versus observation in heavy smokers: 5-year results of the MILD trial. Eur. J. Cancer Prev. 2012, 21, 308-315. [CrossRef] 
9. Saghir, Z.; Dirksen, A.; Ashraf, H.; Bach, K.S.; Brodersen, J.; Clementsen, P.F.; Døssing, M.; Hansen, H.; Kofoed, K.F.; Larsen, K.R.; et al. CT screening for lung cancer brings forward early disease. The randomised Danish lung cancer screening trial: Status after five annual screening rounds with low-dose CT. Thorax 2012, 67, 296-301. [CrossRef]

10. Ortea, I.; Rodríguez-Ariza, A.; Chicano-Gálvez, E.; Arenas Vacas, M.S.; Jurado Gámez, B. Discovery of potential protein biomarkers of lung adenocarcinoma in bronchoalveolar lavage fluid by SWATH MS data-independent acquisition and targeted data extraction. J. Proteom. 2016, 138, 106-114. [CrossRef]

11. Davis, M.D.; Montpetit, A.J. Exhaled Breath Condensate: An Update. Immunol. Allergy Clin. N. Am. 2018, 38, 667-678. [CrossRef]

12. Bruno, D.L.; Haroution, A.; Magda, C.C.; Radu, I. Smell, Lung Cancer, Electronic Nose and Trained Dogs. J. Lung Pulm. Respir. Res. 2014, 1, 47-49. [CrossRef]

13. Phillips, M.; Cataneo, R.N.; Cummin, A.R.C.; Gagliardi, A.J.; Gleeson, K.; Greenberg, J.; Maxfield, R.A.; Rom, W.N. Detection of lung cancer with volatile markers in the breath. Chest 2003, 123, 2115-2123. [CrossRef]

14. Phillips, M.; Bauer, T.L.; Cataneo, R.N.; Lebauer, C.; Mundada, M.; Pass, H.I.; Ramakrishna, N.; Rom, W.N.; Vallières, E. Blinded Validation of Breath Biomarkers of Lung Cancer, a Potential Ancillary to Chest CT Screening. PLoS ONE 2015, 10, e0142484. [CrossRef] [PubMed]

15. Youssef, O.; Sarhadi, V.K.; Armengol, G.; Piirilä, P.; Knuuttila, A.; Knuutila, S. Exhaled breath condensate as a source of biomarkers for lung carcinomas. A focus on genetic and epigenetic markers-A mini-review. Genes Chromosom. Cancer 2016, 55, 905-914. [CrossRef] [PubMed]

16. Mehta, A.; Cordero, J.; Dobersch, S.; Romero-Olmedo, A.J.; Savai, R.; Bodner, J.; Chao, C.; Fink, L.; Guzmán-Díaz, E.; Singh, I.; et al. Non-invasive lung cancer diagnosis by detection of GATA 6 and NKX 2-1 isoforms in exhaled breath condensate. EMBO Mol. Med. 2016, 8, 1380-1389. [CrossRef]

17. López-Sánchez, L.M.; Jurado-Gámez, B.; Feu-Collado, N.; Valverde, A.; Cañas, A.; Fernández-Rueda, J.L.; Aranda, E.; Rodríguez-Ariza, A. Exhaled breath condensate biomarkers for the early diagnosis of lung cancer using proteomics. Am. J. Physiol. Lung Cell. Mol. Physiol. 2017, 313, L664-L676. [CrossRef] [PubMed]

18. Filipowicz, W.; Bhattacharyya, S.N.; Sonenberg, N. Mechanisms of post-transcriptional regulation by microRNAs: Are the answers in sight? Nat. Rev. Genet. 2008, 9, 102-114. [CrossRef]

19. Alipoor, S.D.; Adcock, I.M.; Garssen, J.; Mortaz, E.; Varahram, M.; Mirsaeidi, M.; Velayati, A. The roles of miRNAs as potential biomarkers in lung diseases. Eur. J. Pharmacol. 2016, 791, 395-404. [CrossRef] [PubMed]

20. Carvalho, B.; Bengtsson, H.; Speed, T.P.; Irizarry, R.A. Exploration, normalization, and genotype calls of high-density oligonucleotide SNP array data. Biostatistics 2007, 8, 485-499. [CrossRef]

21. Ritchie, M.E.; Phipson, B.; Wu, D.; Hu, Y.; Law, C.W.; Shi, W.; Smyth, G.K. Limma powers differential expression analyses for RNA-sequencing and microarray studies. Nucleic Acids Res. 2015, 43, e47. [CrossRef] [PubMed]

22. Pinkerton, M.; Chinchilli, V.; Banta, E.; Craig, T.; August, A.; Bascom, R.; Cantorna, M.; Harvill, E.; Ishmael, F.T. Differential expression of microRNAs in exhaled breath condensates of patients with asthma, patients with chronic obstructive pulmonary disease, and healthy adults. J. Allergy Clin. Immunol. 2013, 132, 217-219.e2. [CrossRef]

23. Sinha, A.; Yadav, A.K.; Chakraborty, S.; Kabra, S.K.; Lodha, R.; Kumar, M.; Kulshreshtha, A.; Sethi, T.; Pandey, R.; Malik, G.; et al. Exosome-enclosed microRNAs in exhaled breath hold potential for biomarker discovery in patients with pulmonary diseases. J. Allergy Clin. Immunol. 2013, 132. [CrossRef]

24. Mozzoni, P.; Banda, I.; Goldoni, M.; Corradi, M.; Tiseo, M.; Acampa, O.; Balestra, V.; Ampollini, L.; Casalini, A.; Carbognani, P.; et al. Plasma and EBC microRNAs as early biomarkers of non-small-cell lung cancer. Biomarkers 2013, 18, 679-686. [CrossRef]

25. Zhang, Y.K.; Zhu, W.Y.; He, J.Y.; Chen, D.D.; Huang, Y.Y.; Le, H.B.; Liu, X.G. MiRNAs expression profiling to distinguish lung squamous-cell carcinoma from adenocarcinoma subtypes. J. Cancer Res. Clin. Oncol. 2012, 138, 1641-1650. [CrossRef]

26. Bishop, J.A.; Benjamin, H.; Cholakh, H.; Chajut, A.; Clark, D.P.; Westra, W.H. Accurate classification of non-small cell lung carcinoma using a novel microRNA-based approach. Clin. Cancer Res. 2010, 16, 610-619. [CrossRef]

27. Aharonov, R.; Lebanony, D.; Benjamin, H.; Gilad, S.; Ezagouri, M.; Dov, A.; Ashkenazi, K.; Gefen, N.; Izraeli, S.; Rechavi, G.; et al. Diagnostic assay based on hsa-miR-205 expression distinguishes squamous from nonsquamous non-small-cell lung carcinoma. J. Clin. Oncol. 2009, 27, 2030-2037. [CrossRef]

28. Nadal, E.; Zhong, J.; Lin, J.; Reddy, R.M.; Ramnath, N.; Orringer, M.B.; Chang, A.C.; Beer, D.G.; Chen, G. A microRNA cluster at 14q32 drives aggressive lung adenocarcinoma. Clin. Cancer Res. 2014, 20, 3107-3117. [CrossRef] [PubMed]

29. Gilad, S.; Lithwick-Yanai, G.; Barshack, I.; Benjamin, S.; Krivitsky, I.; Edmonston, T.B.; Bibbo, M.; Thurm, C.; Horowitz, L.; Huang, Y.; et al. Classification of the four main types of lung cancer using a microRNA-based diagnostic assay. J. Mol. Diagn. 2012, 14, 510-517. [CrossRef]

30. Wu, S.G.; Chang, T.H.; Liu, Y.N.; Shih, J.Y. MicroRNA in lung cancer metastasis. Cancers 2019, 11, 265. [CrossRef]

31. Castro, D.; Moreira, M.; Gouveia, A.M.; Pozza, D.H.; De Mello, R.A. MicroRNAs in lung cancer. Oncotarget 2017, 8, 81679-81685. [CrossRef] [PubMed]

32. Xiao, W.; Zhong, Y.; Wu, L.; Yang, D.; Ye, S.; Zhang, M. Prognostic value of microRNAs in lung cancer: A systematic review and meta-analysis. Mol. Clin. Oncol. 2018, 10. [CrossRef] [PubMed] 